\title{
The impacts of morning, daytime, and nighttime symptoms on disease burden in real-world patients with COPD
}

This article was published in the following Dove Press journal: International Journal of COPD

\author{
Anna Muñoz' \\ Mark Small ${ }^{2}$ \\ Robert Wood ${ }^{3}$ \\ Anna Ribera' \\ Javier Nuevo ${ }^{4}$ \\ 'Formerly of Global Medical Affairs, \\ AstraZeneca PLC, Barcelona, Spain; \\ ${ }^{2}$ Respiratory Research, Adelphi Real \\ World, Macclesfield, UK; ${ }^{3}$ Real World \\ Evidence and Epidemiology, Adelphi \\ Real World, Macclesfield, UK; ${ }^{4}$ Global \\ Medical Affairs, AstraZeneca PLC, \\ Madrid, Spain
}

Background: Respiratory symptoms are increasingly recognized as an important consideration in COPD management. Understanding the links between the time(s) of day symptoms are experienced and overall symptom burden could support personalized management strategies. This real-world study aimed to establish the association between the time of day of symptoms and the burden on patients using validated patient-reported outcomes, health care resource utilization, and physician-perceived impact of COPD on patients' lives.

Materials and methods: Analyses used data from four waves (2012, 2013, 2014, and 2016) of the Respiratory Disease Specific Programme: cross-sectional surveys of patients with COPD in Germany, Italy, Spain, and the UK. Patients were classified by their physicians as having symptoms in the morning (M), daytime (D), and/or nighttime (N) in the 4 weeks before entering the Disease Specific Programme. Outcomes included health care resource utilization, work productivity and activity impairment, COPD Assessment Test, EuroQol 5-dimension 3-level questionnaire with visual analog scale, and Jenkins Sleep Evaluation Questionnaire.

Results: In total, 8,844 patients were included, and 8,185 had evaluable time-of-day symptom data. Physicians reported that in the previous 4 weeks, $25 \%$ of patients experienced no symptoms, $16 \% \mathrm{D}$ only, $17 \% \mathrm{M} / \mathrm{D}$ only, $6 \% \mathrm{D} / \mathrm{N}$ only, $4 \% \mathrm{M}$, N, or $\mathrm{M} / \mathrm{N}$ only, and $32 \% \mathrm{M} / \mathrm{D} / \mathrm{N}$. In general, patients with $\mathrm{M} / \mathrm{D} / \mathrm{N}$ symptoms utilized more health care resources in the previous 12 months, had more prior exacerbations, and reported worse activity impairment, health status, and sleep than other symptom groups, whereas patients with symptoms at any time of the day utilized more resources, experienced more exacerbations, and reported worse health status than patients with no symptoms during the 4 weeks before entering the survey.

Conclusion: Patients experiencing morning, daytime, and nighttime symptoms experience a greater disease burden than those in other groups. An individualized approach to COPD treatment based on the timing and persistence of symptoms may improve outcomes for these patients.

Keywords: work productivity, health status, health care resource utilization, activity impairment, sleep, real world

\section{Introduction}

Patients with COPD experience a range of respiratory symptoms that can impact daily activities, sleep, and quality of life. These include breathlessness, cough, sputum production, and chest tightness, ${ }^{1}$ with patients reporting breathlessness as the most problematic symptom. ${ }^{2}$ The 2011 major update to the Global initiative for chronic Obstructive Lung Disease (GOLD) report included revised recommendations for assessing COPD that placed a greater emphasis on respiratory symptoms
Correspondence: Robert Wood Adelphi Real World, Adelphi Mill, Grimshaw Lane, Bollington, Macclesfield, SKIO 5JB, UK

Tel +44 I6 25577308

Email robert.wood@adelphigroup.com 
and exacerbation risk. ${ }^{3}$ The next major update in 2017 took this further by removing spirometric assessment of airflow obstruction from the assessment tool. ${ }^{4}$ These new recommendations categorize patients according to symptom burden and exacerbation history to guide decisions on appropriate therapy, whereas spirometric measures of airflow are seen as a separate assessment primarily for diagnosis.

There is a high degree of variation in the symptom burden experienced by different patients, particularly with regard to the time of day these symptoms are experienced and the impact this has on various aspects of health-related quality of life. ${ }^{1,5,6}$ In terms of COPD symptoms, time of day can be broadly separated into three categories - early morning, daytime, and nighttime - each of which can have a distinct impact on patients' lives. Several studies have reported that early morning is the most common time to experience symptoms, ${ }^{1,2,7}$ and severe symptoms at this time of day are associated with difficulties in performing daily activities such as washing and dressing, ${ }^{1,2}$ as well as an increased risk of exacerbations $^{8}$ and more frequent use of rescue medication. ${ }^{7}$ Moreover, morning symptoms have been negatively associated with physical activity. ${ }^{9}$ Respiratory symptoms are known to impact patients' activity levels - breathlessness and muscle fatigue lead to exercise intolerance, which results in a vicious cycle of physical inactivity. ${ }^{10}$ This exercise limitation occurs even in early disease ${ }^{11}$ and leads to a poorer prognosis. ${ }^{12}$ Controlling morning symptoms has, therefore, been suggested to be a priority in COPD management. ${ }^{9}$ Nighttime symptoms, while found to be less prevalent than early-morning or daytime symptoms in most studies, are associated with an increased risk of cardiac comorbidities, COPD exacerbations, and all-cause mortality. ${ }^{13}$ An additional concern with regard to nighttime symptoms is their impact on sleep disturbance, which is associated with wider health implications. ${ }^{14}$

The ASSESS study of 727 patients with stable COPD found that although symptoms were more prevalent in the early morning and daytime than at night, almost two-thirds of patients (63\%) experienced nighttime symptoms. ${ }^{1}$ In addition, this study also determined that $80 \%$ of patients experienced symptoms in at least two parts of the day and $57 \%$ had symptoms in all three periods. Furthermore, patients experiencing symptoms in more than one part of the day had poorer health status than those with symptoms in only one part of the day, and those experiencing symptoms in all three parts of the day had more anxiety, depression, and sleep disturbance than those with symptoms in one or two parts of the day. ${ }^{1}$

Thus, the time of day in which patients experience COPD symptoms may have a considerable influence over the symptom burden and overall health status. A better understanding of the time of day symptoms occur and of the impact of these symptoms on patients' lives may enable the development of improved and more personalized management strategies and ultimately benefit patients' quality of life. Although the presence of symptoms at particular times of the day has previously been shown to affect disease burden, few studies have investigated the full range of specific impacts contributing to overall disease burden when patients experience symptoms across the entire $24 \mathrm{~h}$ period, as compared with those who experience infrequent symptoms or symptoms in only one or two parts of the day. Therefore, the aim of this novel analysis of survey-based data was to evaluate the association between different timing patterns of symptoms (during one, two, and multiple parts of the day) and the burden experienced by patients, as measured by health care resource utilization (HCRU) and several validated patientreported outcomes (PROs).

\section{Materials and methods Study design}

The Disease Specific Programme (DSP) is a point-in-time survey of physicians and their matched patients, which aims to provide insights into real-world clinical practice across multiple countries for a range of chronic diseases. The general methodology used in the survey has been described previously.$^{15}$ Briefly, a geographically representative sample of eligible physicians was asked to provide information on their next five to six eligible patients by completing an interview and a patient record form. The patients were also invited to complete a patient self-completion form immediately after consultation. Consenting patients responded to the survey confidentially. Unique identification numbers assigned to physicians and patients permitted linked analysis of the completed surveys. Physician- and patient-completed forms were collected by local fieldworkers, with the identifying information removed before submission of surveys for analysis.

Data for this analysis were taken from four waves (2012, 2013, 2014, and 2016) of the Respiratory DSP, from patients with COPD in Germany, Italy, Spain, and the UK, and stratified into novel groups based on whether the COPD symptoms were experienced in the early morning, daytime, and/or nighttime.

\section{Study subjects}

Primary care physicians (PCPs) and pulmonologists were eligible to participate in the Respiratory DSP if they qualified as a physician between 5 and 35 years ago and were 
responsible for the treatment of COPD. Eligible patients were $>40$ years of age, with confirmed airflow obstruction and a diagnosis of COPD (including emphysema and chronic bronchitis). Patients with a concomitant diagnosis of asthma were excluded.

\section{Classification of time of day of symptoms}

For the purposes of this analysis, patients were classified according to the time or times of day symptoms were experienced within the previous 4 weeks, as reported by the physician, based on their judgment following consultation with the patient. The time of day was classified as early morning (defined as "first thing in the morning", "on awakening", and "immediately on awakening" in the 2012 , 2013, and 2014/2016 DSP waves, respectively); daytime (defined as "daytime", "during the day", "during the morning/during the rest of the day", and "during the rest of the day" in the 2012, 2013, 2014, and 2016 DSP waves, respectively); nighttime (defined as "nighttime", "during the night", and "overnight" in the 2012, 2013/2014, and 2016 DSP waves, respectively [the terms "last thing in the evening", "at patient's bedtime", and "during the evening" in the 2012, 2013, and 2016 DSP waves were excluded]); or combinations of these. In defining whether patients experienced symptoms in each time period, responses of "once or twice a week", "3-6 times a week", and "every day" were included and responses of "not at all" and "less than once a week" were excluded. Patients with responses of "not at all" or "less than once a week" for all three time periods in the previous 4 weeks were classified as having no symptoms. Due to relatively few patients experiencing symptoms in the morning and/or nighttime, these categories were pooled into a single group. Therefore, the six categories used in this analysis were: no symptoms; daytime only (D only); morning and daytime only (M/D only); daytime and nighttime only (D/N only); morning, nighttime, or morning and nighttime only $(\mathrm{M}, \mathrm{N}$, or $\mathrm{M} / \mathrm{N}$ only); or morning, daytime, and nighttime $(\mathrm{M} / \mathrm{D} / \mathrm{N})$.

\section{Outcome variables}

Outcome variables were either recorded directly by the patient or physician or derived from the patient record forms or patient self-completion forms. Descriptive variables included age, sex, body mass index, smoking status, most recent postbronchodilator forced expiratory volume in 1 second (FEV $1 \%$ predicted), and GOLD (2017) group (calculated from collected variables). ${ }^{4} \mathrm{HCRU}$ data, including number of physician visits for COPD, number of exacerbations requiring hospitalization, and cumulative number of inpatient nights in the 12 months prior to the survey, were provided by the physicians. Other outcomes were recorded using a series of validated PRO tools: activity impairment was assessed using the Work Productivity and Activity Impairment: Specific Health Problem (WPAI:SHP) questionnaire, ${ }^{16}$ adapted for COPD; impact of COPD symptoms was assessed using the COPD Assessment Test (CAT); ${ }^{17}$ health status was evaluated using the EuroQol 5-dimension 3-level questionnaire (EQ-5D-3L) and a standard vertical $20 \mathrm{~cm}$ visual analog scale (EQ-VAS); ${ }^{18}$ and sleep quality was assessed using the Jenkins Sleep Evaluation Questionnaire (JSEQ). ${ }^{19}$

\section{Statistical analysis}

Patient demographics and clinical characteristics were summarized by descriptive statistics. Kruskal-Wallis tests were used to determine the statistical significance of outcomes across patient groups and to provide a nominal $p$-value, and Bonferroni-adjusted Mann-Whitney $U$-tests were used to determine the statistical significance of differences in outcomes between pairs of patient groups. All analyses were performed in Stata v14 (StataCorp LLC, College Station, TX, USA).

\section{Ethics approval and informed consent}

The research was conducted as a market research survey in accordance with the amended Declaration of Helsinki, adhering to the International Chamber of Commerce/European Society for Opinion and Marketing Research code on observational research. Institutional review board approval was, therefore, not required or sought.

Patients anonymously provided their consent to participate in this study by checking a box to confirm their consent after having read information about the research and the subsequent use of data.

\section{Results}

\section{Study population}

In total, 607 physicians and 8,844 patients were included across the four waves (2,176 from 2012, 2,180 from 2013, 2,209 from 2014, and 2,279 from 2016). The four countries were evenly represented in terms of patient numbers, with 2,283 patients from Germany, 2,255 from Italy, 2,285 from Spain, and 2,021 from the UK. Of the 8,185 patients who had evaluable data for the time-of-day symptom analysis, $48.5 \%$ were recruited by PCPs and $51.5 \%$ by pulmonologists. 
Patient demographics and clinical characteristics are summarized in Table 1 . The majority of patients were male (69.2\%), and 66.6\% were ex-smokers. Patients were most likely to be categorized as GOLD (2017) group B (53.2\%) or $\mathrm{D}(35.5 \%)$, suggesting that most patients in this sample were symptomatic. Patient characteristics were similar across all four waves (data not shown). Most patients ( $\geq 94 \%$ ) were receiving at least one maintenance therapy for COPD, including long-acting $\beta_{2}$-agonists (LABA), long-acting muscarinic antagonists (LAMA), and/or inhaled corticosteroids (ICS). The most commonly prescribed maintenance regimens were ICS+LABA+LAMA (in two or three devices; 37.5\%), LAMA alone (20.6\%), ICS+LABA (in one or two devices; 16.4\%), LABA+LAMA (in one or two devices; 11.8\%), and LABA alone (4.7\%), as shown in Table 1. Most patients reported either $\mathrm{M} / \mathrm{D} / \mathrm{N}$ symptoms (32.4\%) or no symptoms (25.2\%) in the previous 4 weeks (Figure 1). Across the four waves,

Table I Patient demographics and clinical characteristics ${ }^{\mathrm{a}}$

\begin{tabular}{|c|c|}
\hline & $\begin{array}{l}\text { Overall } \\
(\mathrm{N}=\mathbf{8 , 8 4 4})\end{array}$ \\
\hline \multicolumn{2}{|l|}{ DSP wave, $n(\%)$} \\
\hline 2012 & $2,176(24.6)$ \\
\hline 2013 & $2,180(24.6)$ \\
\hline 2014 & $2,209(25.0)$ \\
\hline 2016 & $2,279(25.8)$ \\
\hline Mean age, years $(S D)^{b}$ & $66.3(10.3)$ \\
\hline Male, n (\%) & $6,115(69.2)$ \\
\hline Mean body mass index, $\mathrm{kg} / \mathrm{m}^{2}$ (SD) & $26.6(4.6)$ \\
\hline \multicolumn{2}{|l|}{ Smoking status, n (\%) } \\
\hline Current smoker & $2,763(33.4)$ \\
\hline Ex-smoker & $5,5 \mid 4(66.6)$ \\
\hline Mean postbronchodilator $\mathrm{FEV}_{1}$, \% predicted (SD) & $62.3(17.7)$ \\
\hline \multicolumn{2}{|l|}{ GOLD group (2017), n (\%) } \\
\hline A & $468(10.3)$ \\
\hline B & $2,419(53.2)$ \\
\hline $\mathrm{C}$ & $47(1.0)$ \\
\hline $\mathrm{D}$ & $1,615(35.5)$ \\
\hline \multicolumn{2}{|l|}{ Current prescribed therapy, $\mathrm{n}(\%)$} \\
\hline SABA, SAMA, or SABA/SAMA & $332(3.8)$ \\
\hline LABA alone & $409(4.7)$ \\
\hline LAMA alone & $\mathrm{I}, 798(20.6)$ \\
\hline ICS alone & $190(2.2)$ \\
\hline $\mathrm{ICS}+\mathrm{LABA}^{\mathrm{c}}$ & $\mathrm{I}, 43 \mathrm{I}(16.4)$ \\
\hline ICS+LAMA ${ }^{d}$ & $88(1.0)$ \\
\hline LABA+LAMA ${ }^{c}$ & $1,029(11.8)$ \\
\hline $\mathrm{ICS}+\mathrm{LABA}+\mathrm{LAMA}^{\mathrm{e}}$ & $3,264(37.5)$ \\
\hline Other & $172(2.0)$ \\
\hline
\end{tabular}

Notes: ${ }^{a}$ Data shown are for patients with evaluable data for each characteristic. bFor calculation of descriptive statistics, patients reported to be $>90$ years of age $(0.8 \%)$ were assumed to be 90 years of age, in order to protect patient identity. In one or two devices. IIn two devices. eln two or three devices.

Abbreviations: DSP, Disease Specific Programme; $\mathrm{FEV}_{1}$, forced expiratory volume in I second; GOLD, Global initiative for chronic Obstructive Lung Disease; ICS, inhaled corticosteroid; LABA, long-acting $\beta_{2}$-agonist; LAMA, long-acting muscarinic antagonist; SABA, short-acting $\beta_{2}$-agonist; SAMA, short-acting muscarinic antagonist.

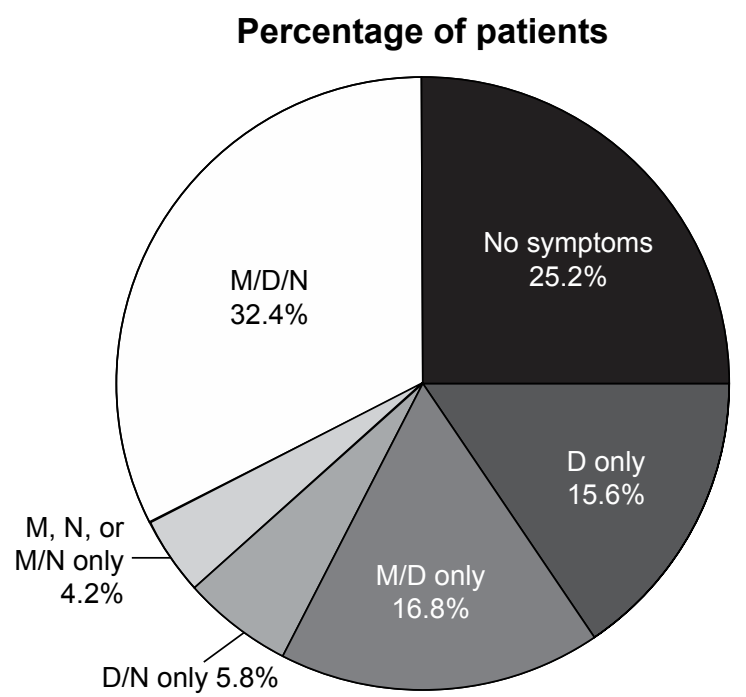

Figure I Percentage of patients experiencing symptoms (previous 4 weeks), by time of day of symptoms.

Abbreviations: $D$ only, daytime only; D/N only, day and nighttime only; M/D only, morning and daytime only; $M / D / N$, morning, daytime, and nighttime; $M, N$, or $M / N$ only, morning or nighttime, or morning and nighttime only.

the percentage of patients suffering any $\mathrm{M}, \mathrm{D}$, or $\mathrm{N}$ symptoms ranged between $44 \%$ and $63 \%, 67 \%$ and $77 \%$, and $36 \%$ and $45 \%$, respectively.

\section{Health care resource utilization}

The mean numbers of COPD-related PCP and pulmonologist consultations in the last 12 months by time of day of symptoms are presented in Figure 2A and B, respectively. In the 12 months prior to the survey, patients experiencing $\mathrm{D}$ only, $\mathrm{D} / \mathrm{N}$ only, and $\mathrm{M} / \mathrm{D} / \mathrm{N}$ symptoms visited PCPs significantly more frequently than patients experiencing no symptoms (3.1, 3.8, and 4.4 visits vs 2.8 visits, respectively; all $p<0.05$; Figure $2 \mathrm{~A}$ ). Additionally, patients with $\mathrm{D} / \mathrm{N}$ only or $\mathrm{M} / \mathrm{D} / \mathrm{N}$ symptoms visited PCPs significantly more frequently than patients with symptoms at other times of the day (all $p<0.001$; Figure 2A). Patients experiencing D only, M/D only, D/N only, and $\mathrm{M} / \mathrm{D} / \mathrm{N}$ symptoms visited pulmonologists significantly more frequently (2.1-2.8 visits) than patients experiencing no symptoms (1.7 visits; all $p<0.001$; Figure 2B). Furthermore, patients experiencing $\mathrm{M} / \mathrm{D} / \mathrm{N}$ symptoms visited pulmonologists significantly more frequently than any other group of patients ( 2.8 visits), including patients with $\mathrm{D} / \mathrm{N}$ only symptoms (all $p<0.005$; Figure $2 \mathrm{~B}$ ).

The mean numbers of COPD-related exacerbations leading to emergency room visits or hospital admissions in the last 12 months by the time of symptoms are shown in Figure $3 \mathrm{~A}$ and $\mathrm{B}$, respectively. In the 12 months prior to the survey, patients experiencing symptoms at any time 

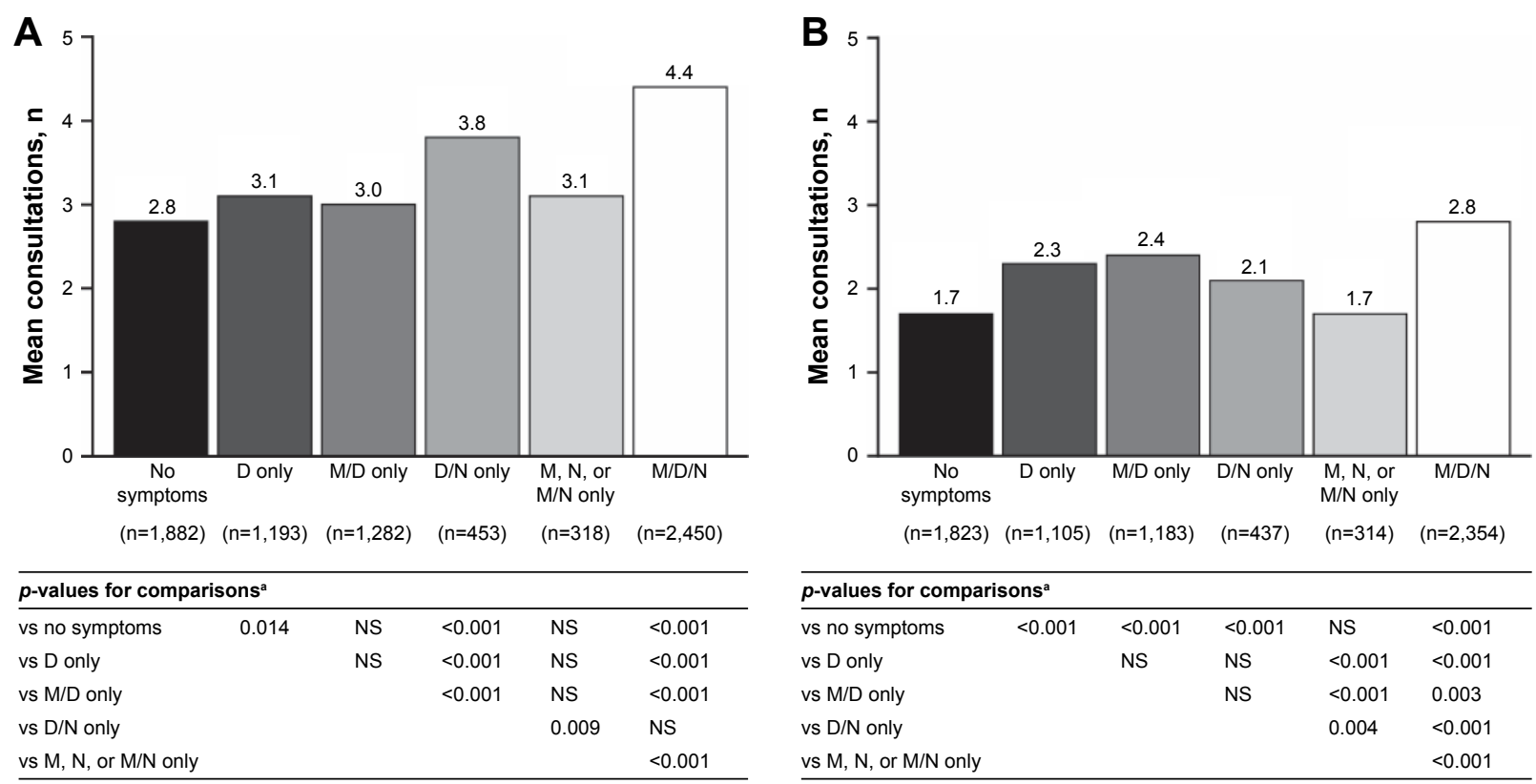

Figure 2 Mean number of (A) PCP consultations or (B) pulmonologist consultations (last 12 months).

Note: ${ }^{a} p$-values for comparisons generated by Bonferroni-adjusted Mann-Whitney U-test.

Abbreviations: $D$ only, daytime only; D/N only, day and nighttime only; M/D only, morning and daytime only; M/D/N, morning, daytime, and nighttime; $M$, N, or M/N only, morning or nighttime, or morning and nighttime only; NS, nonsignificant; PCP, primary care physician.

of the day (D only; M/D only; D/N only; $\mathrm{M}, \mathrm{N}$, or $\mathrm{M} / \mathrm{N}$ only; and $\mathrm{M} / \mathrm{D} / \mathrm{N}$ ) had significantly more exacerbations requiring emergency room visits $(0.1-0.3)$ or leading to hospital admissions $(0.1-0.5)$ than patients experiencing no symptoms (both 0.0 ; all $p<0.001$; Figure 3 ). Additionally, patients experiencing $\mathrm{M} / \mathrm{D} / \mathrm{N}$ symptoms had significantly more exacerbations requiring emergency room visits or leading to hospital admissions ( 0.3 and 0.5 , respectively) than any other group of patients (all $p<0.001$ ), with the exception of exacerbations requiring emergency room visits
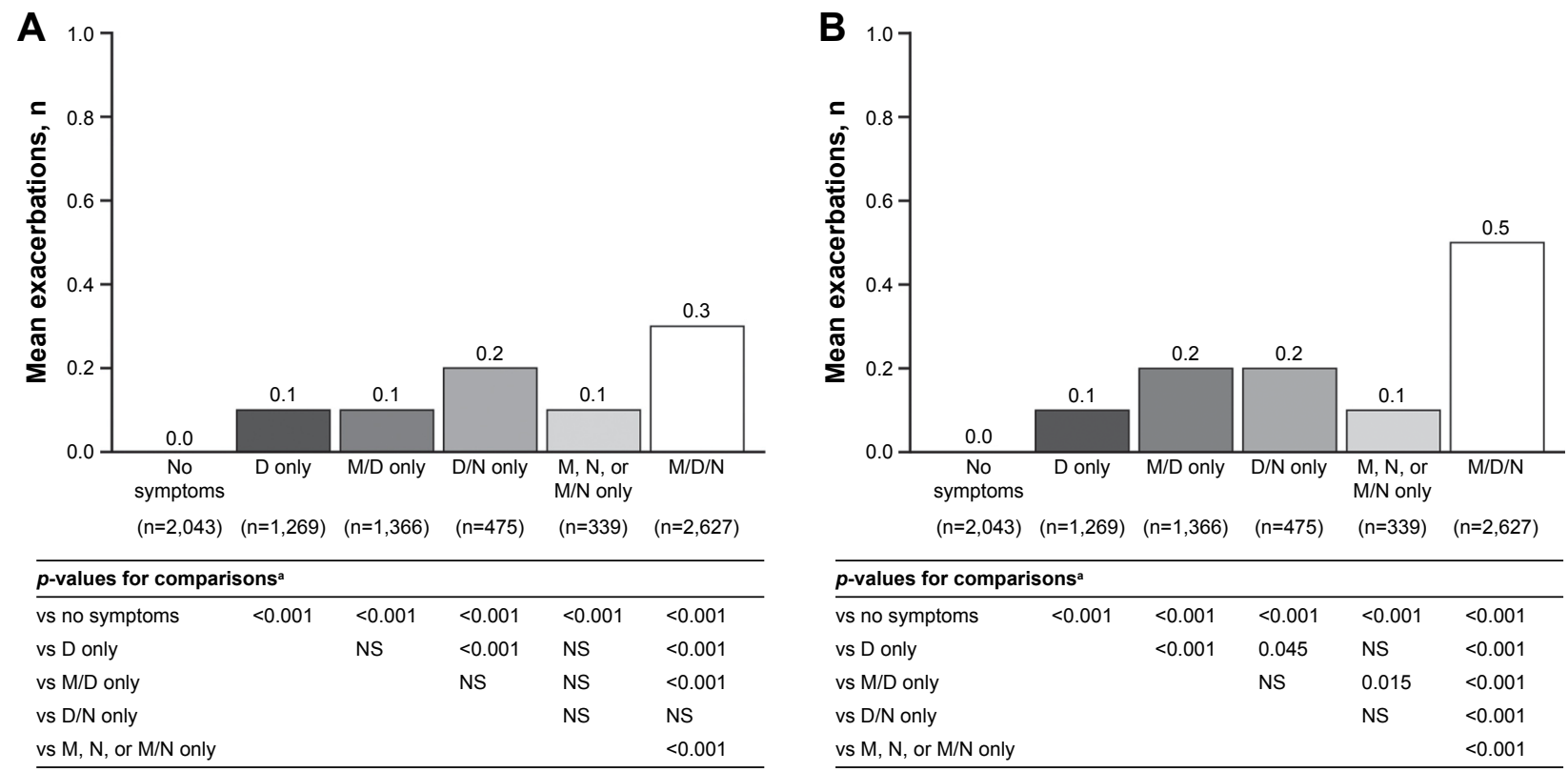

Figure 3 Mean exacerbations leading to (A) ER visits or (B) hospital admissions (last 12 months).

Note: a $p$-values for comparisons generated by Bonferroni-adjusted Mann-Whitney U-test.

Abbreviations: $\mathrm{D}$ only, daytime only; $\mathrm{D} / \mathrm{N}$ only, day and nighttime only; $E R$, emergency room; $M / D$ only, morning and daytime only; $M / D / N$, morning, daytime, and nighttime; $\mathrm{M}, \mathrm{N}$, or $\mathrm{M} / \mathrm{N}$ only, morning or nighttime, or morning and nighttime only; NS, nonsignificant. 
in $\mathrm{D} / \mathrm{N}$ only patients, where the difference was not significant ( $p=0.085$; Figure 3$)$.

The mean cumulative numbers of inpatient nights and nights in intensive care due to exacerbations are shown in Figure $4 \mathrm{~A}$ and $\mathrm{B}$, respectively. In the 12 months prior to the survey, patients experiencing symptoms at any time of the day had more inpatient nights as a result of exacerbations than patients experiencing no symptoms (all $p<0.001$; Figure 4A). Patients with M/D only, D/N only, or M/D/N symptoms spent more nights in intensive care than patients experiencing no symptoms (all $p<0.05$; Figure 4B). Furthermore, patients experiencing $\mathrm{M} / \mathrm{D} / \mathrm{N}$ symptoms had more inpatient and intensive-care-unit nights than any other group of patients (all $p<0.001$; Figure 4).

\section{PROs}

\section{Work productivity and activity impairment}

Percentage overall work impairment and percentage activity impairment by the time of symptoms are shown in Figure 5A and $\mathrm{B}$, respectively. Work impairment-related questions in the WPAI:SHP questionnaire were answered by employed patients only, resulting in smaller patient numbers for this outcome. Patients experiencing D only, M/D only, D/N only, or $\mathrm{M} / \mathrm{D} / \mathrm{N}$ symptoms reported significantly higher mean overall work impairment $(25.5 \%-41.2 \%)$ than patients experiencing no symptoms (16.2\%; all $p<0.001$; Figure $5 \mathrm{~A}$ ). Patients experiencing $\mathrm{M} / \mathrm{D} / \mathrm{N}$ symptoms reported the highest mean overall work impairment (41.2\%), which, with the exception of $\mathrm{D} / \mathrm{N}$ only, was significantly higher than any other symptom group $(p<0.001)$. Additionally, patients with $\mathrm{D} / \mathrm{N}$ only symptoms reported a mean overall work impairment of $37.2 \%$, which was significantly higher than that of patients with D only or M, N, or M/N only symptoms (both $p<0.05$ ). Activity impairment followed a similar pattern; patients experiencing symptoms at any time of the day reported significantly higher mean activity impairment $(33.7 \%-56.4 \%)$ than patients experiencing no symptoms (24.6\%; all $p<0.001$; Figure 5B). Patients experiencing $\mathrm{M} / \mathrm{D} / \mathrm{N}$ symptoms, who reported the highest level of activity impairment (56.4\%), had significantly higher mean activity impairment than any other symptom group (all $p<0.001$; Figure 5B). Additionally, patients with $\mathrm{D} / \mathrm{N}$ only symptoms reported a mean activity impairment of $45.0 \%$, which was significantly higher than that reported by patients experiencing $\mathrm{D}$ only or $\mathrm{M}, \mathrm{N}$, or $\mathrm{M} / \mathrm{N}$ only symptoms (both $p<0.001$; Figure $5 \mathrm{~B}$ ).

\section{Health status and impact of COPD symptoms}

Patients experiencing symptoms at any time of the day had significantly higher mean CAT scores (18.4-25.7) than those patients experiencing no symptoms (14.2; all $p<0.001$; Figure 6). Furthermore, patients experiencing $\mathrm{M} / \mathrm{D} / \mathrm{N}$ symptoms had the highest mean CAT score (25.7), which was significantly higher than any other symptom group (18.4-21.5; all $p<0.001$; Figure 6). Additionally, patients
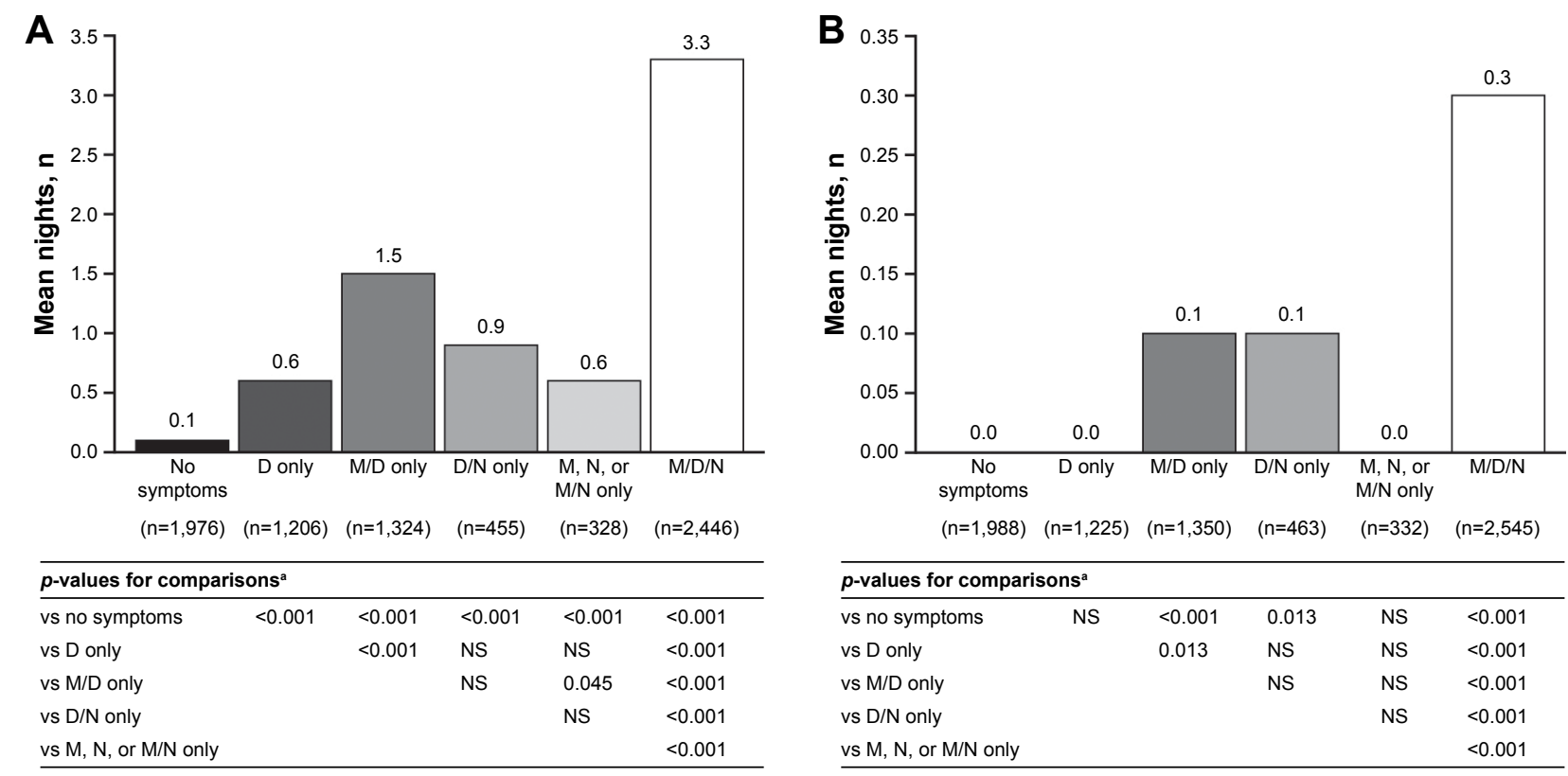

Figure 4 Mean cumulative $(\mathbf{A})$ inpatient nights and $(\mathbf{B})$ intensive care nights as a result of exacerbations.

Note: a $p$-values for comparisons generated by Bonferroni-adjusted Mann-Whitney U-test.

Abbreviations: $\mathrm{D}$ only, daytime only; D/N only, day and nighttime only; M/D only, morning and daytime only; M/D/N, morning, daytime, and nighttime; $M$, N, or M/N only, morning or nighttime, or morning and nighttime only; NS, nonsignificant. 

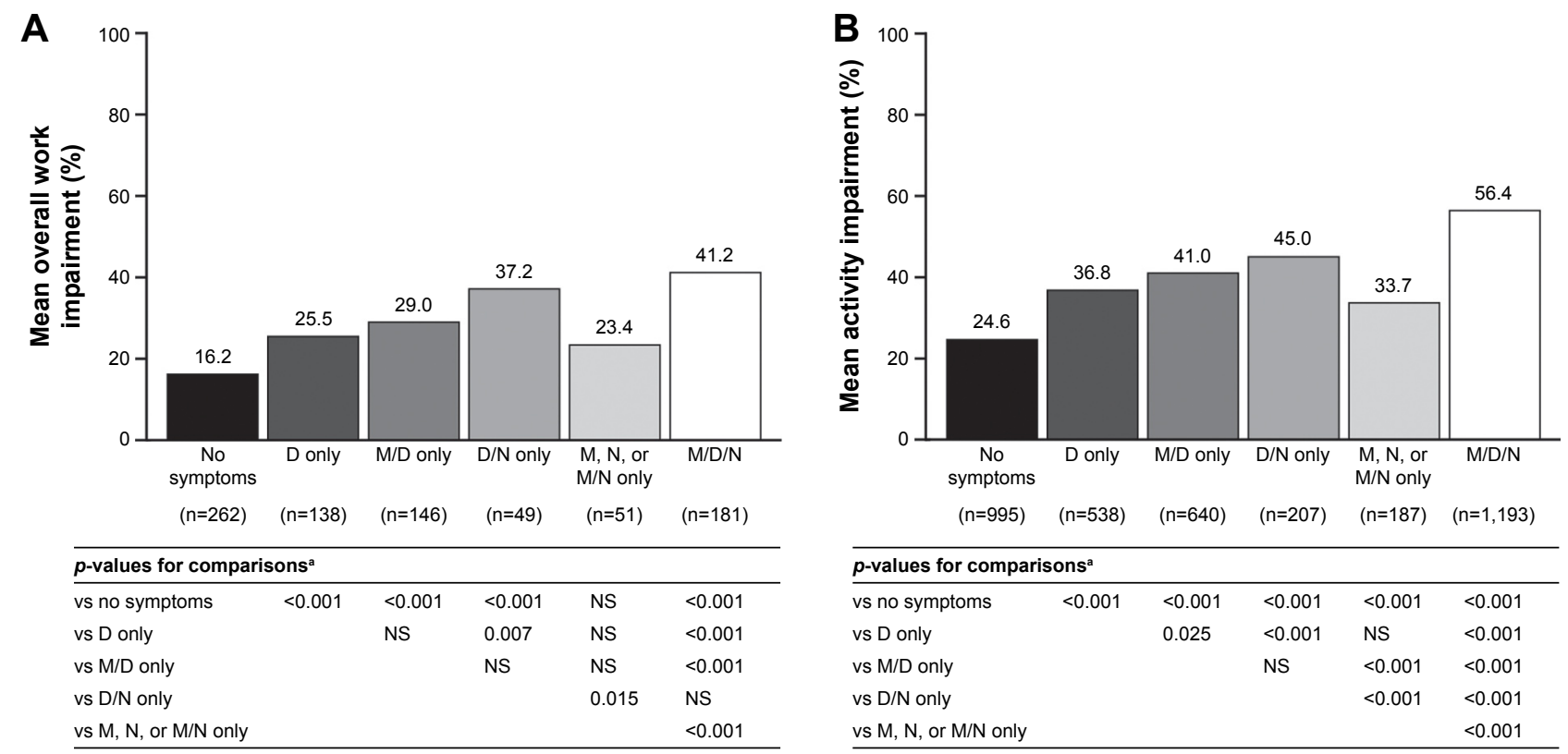

Figure 5 Mean percentage of $(\mathbf{A})$ overall work impairment and $(\mathbf{B})$ activity impairment as measured by WPAl:SHP questionnaire. ${ }^{b}$

Notes: ${ }^{a} p$-values for comparisons generated by Bonferroni-adjusted Mann-Whitney U-test. ${ }^{b}$ Work impairment-related questions were answered by employed patients only, resulting in a smaller patient base.

Abbreviations: $D$ only, daytime only; D/N only, day and nighttime only; M/D only, morning and daytime only; M/D/N, morning, daytime, and nighttime; $M$, N, or M/N only, morning or nighttime, or morning and nighttime only; NS, nonsignificant; WPAl:SHP, Work Productivity and Activity Impairment: Specific Health Problem.

experiencing $\mathrm{D} / \mathrm{N}$ only symptoms had a mean CAT score of 21.5, which was significantly higher than the mean CAT scores of patients experiencing D only or $\mathrm{M}, \mathrm{N}$, or M/N only symptoms (both $p<0.001$; Figure 6).

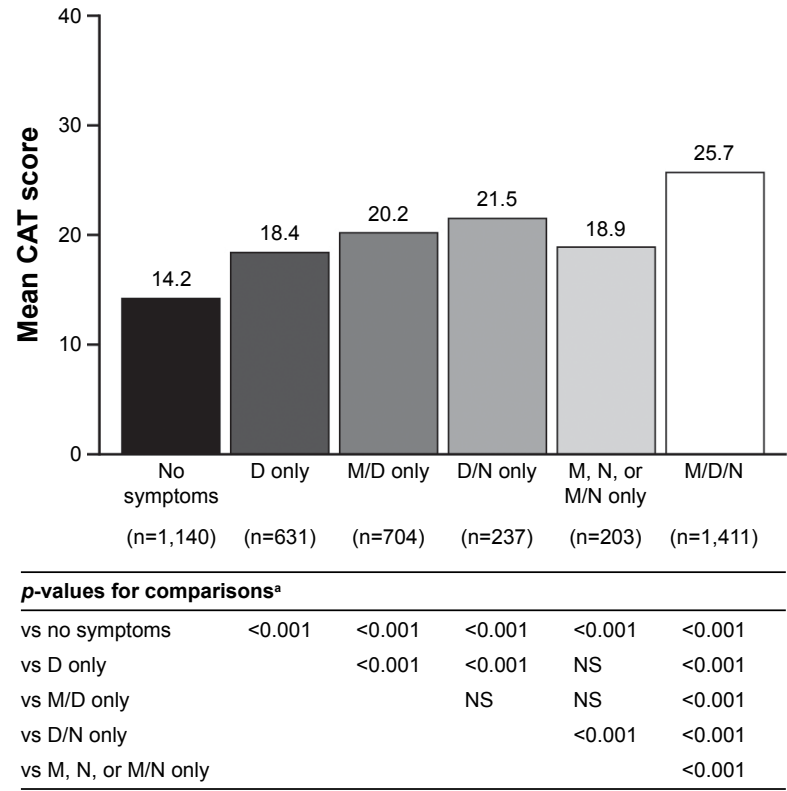

Figure 6 Mean CAT score.

Note: ${ }^{a} p$-values for comparisons generated by Bonferroni-adjusted Mann-Whitney U-test.

Abbreviations: CAT, COPD Assessment Test; D only, daytime only; D/N only, day and nighttime only; M/D only, morning and daytime only; $M / D / N$, morning, daytime, and nighttime; $\mathrm{M}, \mathrm{N}$, or $\mathrm{M} / \mathrm{N}$ only, morning or nighttime, or morning and nighttime only; NS, nonsignificant.
Health status, as measured by EQ-5D-3L and EQ-VAS (Figure 7A and B, respectively) followed a similar pattern to CAT score. Patients with symptoms at any time of the day reported lower scores (EQ-5D-3L: 0.69-0.86; EQ-VAS: 56.3-68.6) than patients experiencing no symptoms (EQ-5D-3L: 0.89; EQ-VAS: 72.7; all $p<0.05$; Figure 7). Once again, patients with $\mathrm{M} / \mathrm{D} / \mathrm{N}$ symptoms reported the worst scores (EQ-5D-3L: 0.69; EQ-VAS: 56.3), which were significantly lower than those reported by patients in any other symptom group (all $p<0.001$; Figure 7).

\section{Sleep quality}

Patients experiencing symptoms at any time of the day had poorer sleep quality, as indicated by higher JSEQ scores (3.6-8.3), than patients with no symptoms (2.3; all $p<0.001$; Figure $8 \mathrm{~A}$ ). Furthermore, patients with $\mathrm{M} / \mathrm{D} / \mathrm{N}$ symptoms had the highest mean JSEQ score (8.3), which was significantly higher than that of patients in any other symptom group (all $p<0.001$; Figure 8A). Additionally, patients experiencing $\mathrm{D} / \mathrm{N}$ only symptoms had a JSEQ score of 5.6, which was significantly higher than that of patients with D only, M/D only, or $\mathrm{M}, \mathrm{N}$, or $\mathrm{M} / \mathrm{N}$ only symptoms (all $p<0.001$; Figure $8 \mathrm{~A}$ ). When considering the impact of COPD on their sleep, the impact reported by patients experiencing $\mathrm{M} / \mathrm{D} / \mathrm{N}$ symptoms was significantly different to that reported by any other symptom group (all $p<0.001$ ), with $71 \%$ of $\mathrm{M} / \mathrm{D} / \mathrm{N}$ patients reporting a medium, high, or constant impact (Figure 8B). 

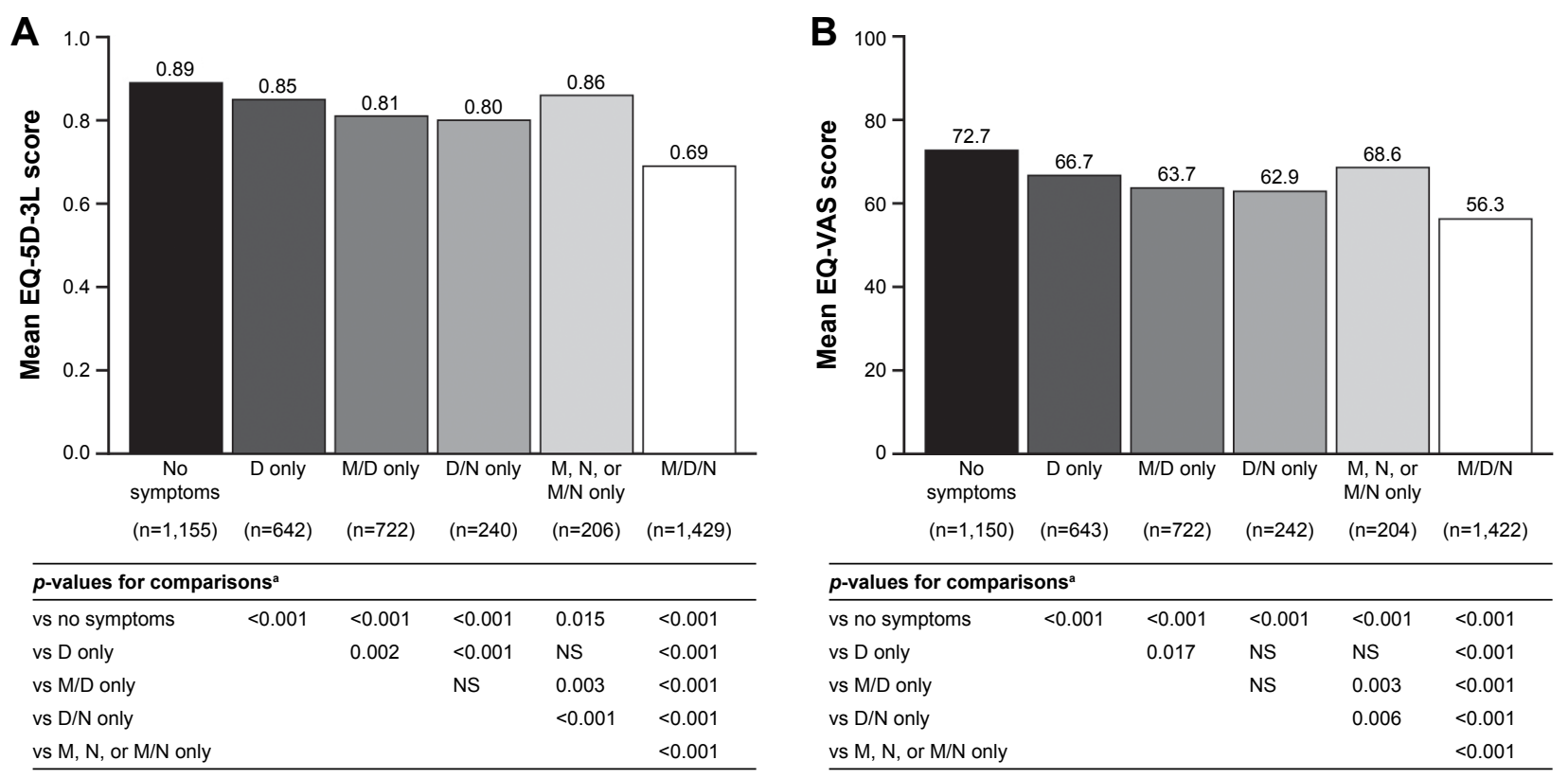

Figure 7 Mean (A) EQ-5D-3L and (B) EQ-VAS scores.

Note: a ${ }^{p}$-values for comparisons generated by Bonferroni-adjusted Mann-Whitney U-test.

Abbreviations: D only, daytime only; D/N only, day and nighttime only; EQ-5D-3L, EuroQol 5-dimension 3-level questionnaire; EQ-VAS, EuroQol visual analog scale; M/D only, morning and daytime only; M/D/N, morning, daytime, and nighttime; $\mathrm{M}, \mathrm{N}$, or M/N only, morning or nighttime, or morning and nighttime only; NS, nonsignificant.

Additionally, $58 \%$ of patients with $\mathrm{D} / \mathrm{N}$ only symptoms reported a medium, high, or constant impact on sleep, compared with $21 \%-39 \%$ of patients in the remaining symptom groups (Figure 8B).

\section{Discussion}

In this analysis of survey-based, real-world data, we found that patients who experienced COPD symptoms at any time of the day over a 4-week period utilized significantly more
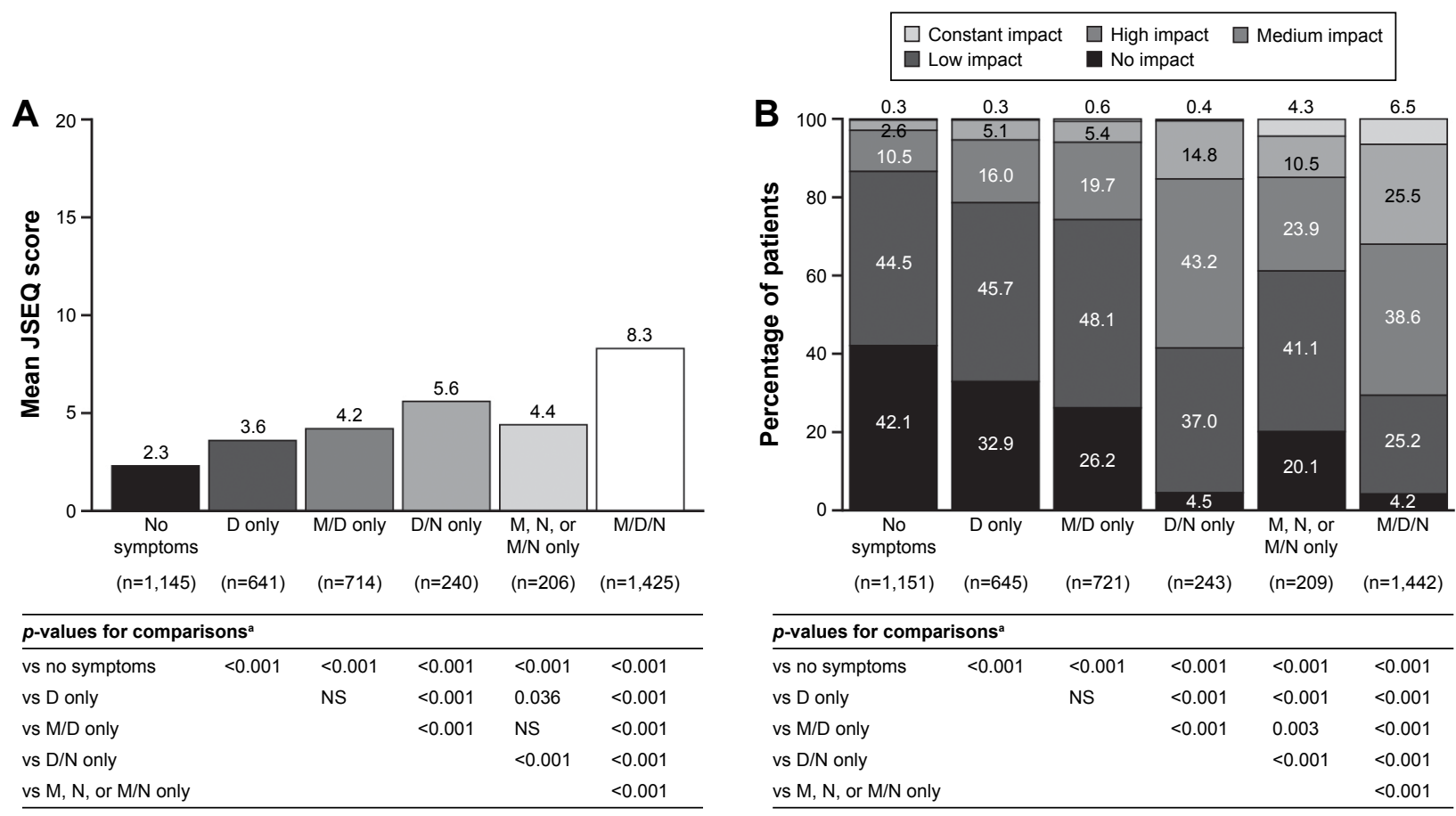

Figure 8 (A) Mean JSEQ score and (B) patient-reported impact of COPD on patients' sleep.

Note: ${ }^{a}$-values for comparisons generated by Bonferroni-adjusted Mann-Whitney U-test.

Abbreviations: D only, daytime only; D/N only, day and nighttime only; JSEQ, Jenkins Sleep Evaluation Questionnaire; M/D only, morning and daytime only; M/D/N, morning, daytime, and nighttime; $\mathrm{M}, \mathrm{N}$, or $\mathrm{M} / \mathrm{N}$ only, morning or nighttime, or morning and nighttime only; NS, nonsignificant. 
health care resources and had more exacerbations in the previous 12 months, experienced greater impairment of work and activity, and had poorer health status and sleep quality than patients with no symptoms in the 4 weeks prior to visiting their physician. Patients experiencing symptoms during all three parts of the day over 4 weeks - in the morning, daytime, and nighttime - utilized significantly more health care resources, and had more exacerbations in the previous 12 months and suffered a greater impact on activity, health status, and sleep quality than patients in all other symptom groups. As such, these patients had the worst disease burden, and therefore stand to benefit most from management strategies that take into account the reduction of symptoms as a treatment goal.

Previous studies have demonstrated that the time of day COPD symptoms are experienced can have different impacts on health status and quality of life..$^{2,7,13}$ However, while we recognize that certain times of the day are associated with specific impacts, we report here that the presence of symptoms during multiple parts of the day, rather than the time of day they occur per se, has a greater overall impact on health outcomes.

We found that patients most commonly experienced symptoms in all three parts of the day, which is consistent with published data from the ASSESS study. ${ }^{1}$ While ASSESS was the first study to demonstrate associations between symptoms in each part of the $24 \mathrm{~h}$ day and outcomes including CAT score, sleep quality, and HCRU, ${ }^{1,20}$ we have confirmed these findings in a much larger population and expanded them to demonstrate that this symptom pattern has additional impacts on work productivity, activity, and health status measured by the EQ-5D-3L. This shows that a large proportion of COPD patients stand to benefit - through potential improvements in many aspects of the COPD disease burden - from a greater recognition of symptom burden in COPD treatment guidelines. Our finding that these patients had greater HCRU in the previous 12 months than other symptom groups (building on the finding from ASSESS that patients with symptoms in all three parts of the day made more visits to PCPs $)^{20}$ suggests that improving $24 \mathrm{~h}$ symptom control could alleviate the economic burden associated with COPD. These data also highlight an association between increased incidence of exacerbations requiring hospital visits and the presence of symptoms during multiple parts of the day, further demonstrating the importance of evaluating the timing of symptoms when deciding management options.

The use of validated PRO tools, such as those used in this analysis, enables quantification of the impact of COPD symptoms on patients in a real-world setting. While breathlessness is considered to be the most characteristic COPD symptom and has historically been used to quantify symptoms, the most recent GOLD guidelines state that a more thorough and detailed assessment of symptoms (including cough, sputum, and chest tightness) and their impact on health status, using tests such as the CAT, is required to adequately quantify the symptom burden. ${ }^{4} \mathrm{We}$ report here that incremental increases in symptom burden in patients with morning, daytime, and nighttime symptoms are reflected by the CAT score, with patients reporting symptoms in all three parts of the day having significantly higher CAT scores than those reporting symptoms in only one or two parts of the day. However, the mean CAT score of patients experiencing no symptoms over a 4-week period in this study was higher than 10 (the cutoff usually used to define low impact of symptoms). This suggests that although these patients' symptoms were apparently controlled and stable over the 4-week period assessed, they nonetheless experience an impact on daily life as a result of their COPD and would be classified as symptomatic according to the GOLD 2017 assessment tool. This may also reflect the fact that certain items in the CAT, namely those related to energy levels and confidence to leave the house, can still impact patients, even though symptoms have stabilized in the short term.

Our results demonstrate that activity limitation and work impairment are two important aspects of the COPD symptom burden that are affected by persistent symptoms. Although morning symptoms are perceived by patients to have the greatest impact on performing specific daily activities, ${ }^{2,6}$ we found that patients experiencing symptoms either in the daytime and nighttime or in all three parts of the day had greater overall impairment of work productivity and activity than patients with symptoms in the morning, nighttime, or morning and nighttime only or in the daytime only. Similarly, two recent papers by Ding et al report that greater frequency and overall burden of COPD symptoms are associated with poorer health status and increased work and activity impairment, ${ }^{21,22}$ and a recent systematic review found morning symptoms to be associated with a sedentary lifestyle and a negative impact on physical activity. ${ }^{9}$ Our analysis builds on this previous evidence to show that the timing pattern of symptoms (whether the symptoms are spread across multiple parts of the day) may have a greater impact on activity limitation than simply the frequency of symptoms or whether symptoms are experienced specifically in the morning.

Activity limitation is a consequence of COPD symptoms that has a severe impact on patients' daily routine and quality of life $;^{8}$ however, evidence shows that patients are unlikely 
to discuss this with their physicians. ${ }^{23}$ Additionally, GOLD 2017 suggests that some patients report minimal symptoms because they are self-limiting their activity in order to avoid triggering symptoms; therefore, although their COPD appears to be well controlled, they would likely benefit from improved management of their COPD. ${ }^{4}$ It will, therefore, be important to give due consideration to this aspect of the COPD symptom burden in the development of personalized treatment approaches.

Sleep disruption may be considered a direct result of nighttime symptoms; however, we found that patients experiencing symptoms in the daytime and nighttime or in all three parts of the day had higher JSEQ scores and perceived a greater impact on their sleep than patients with symptoms in the morning or nighttime, or morning and nighttime only. While this analysis did not monitor symptom severity, experiencing symptoms in all three parts of the day has previously been reported to correlate with more severe dyspnea. ${ }^{1}$ This greater severity of symptoms may account for the increased sleep disruption we observed. The impact of persistent symptoms on sleep is of particular clinical concern, as sleep disruption is associated with worse symptoms, poorer health status, and higher risks of exacerbations and mortality in COPD, as well as increased risks of anxiety and depression and poorer health-related quality of life. ${ }^{14,24-26}$ Sleep impairment has also been associated with reduced daytime physical activity (the implications of which are discussed above), and therefore, managing nighttime symptoms could have far-reaching therapeutic benefits. ${ }^{27}$ It may, therefore, be valuable to evaluate the presence of nighttime symptoms and their potential impact on sleep quality, in order to identify patients with a greater risk of poor outcomes to ensure they receive adequate treatment.

A limitation of this analysis is that the 4-week time period over which symptoms were assessed did not align with the time frame of all outcomes. Although the WPAI:SHP, CAT, health status, and sleep outcomes covered the same period as the physician-reported symptom assessment, HCRU and exacerbations were assessed over a much longer time period of 1 year prior to the physician visit. Consequently, a causal relationship cannot be inferred; however, the results clearly indicate an association between these factors.

Some characteristics of the population recruited to the survey may limit the generalizability to the general COPD patient population. First, the proportion of patients recruited by pulmonologists is likely higher than that generally seen in clinical practice; therefore, the analysis may be biased toward patients with more severe COPD. Second, the analysis population included a high proportion of patients in GOLD groups B and D. Our findings may, therefore, not be fully generalizable to less symptomatic patients or those with less severe disease; however, our conclusion that clinicians should consider the time(s) of day the symptoms occur and manage patients accordingly to reduce the resultant burden applies throughout the general COPD population.

The definitions of morning, daytime, and nighttime in this study reflect the real-world nature of the survey approach, which limited the precision of the time-of-day classifications in this analysis. However, ambiguous responses such as "during the evening" were excluded to make the classifications as precise and valid as possible. Other, more general limitations of the DSP approach have been discussed previously. ${ }^{15}$ The methodology used to select the samples is not truly random, and leaves the survey open to potential participation bias. Similarly, the collection of symptom data depends upon accurate recall and interpretation by the patient and physician, introducing the possibility of inaccuracies. For example, the time-of-day classification is subjective and open to recall bias; although this assessment was based on physician reporting, the information was obtained through consultation with the patient. Equally, patients experiencing severe symptoms may have been more likely to remember them as occurring more persistently, which could have influenced the results. However, with these limitations in mind, the strength of the DSP approach is that it provides a "snapshot" of large numbers of patients with COPD in a real-world clinical setting, increasing the likelihood that conclusions are relevant to the wider population.

\section{Conclusion}

Our results demonstrate that patients who experience symptoms in all three parts of the day - morning, daytime, and nighttime - have worse PROs, greater prior HCRU (implying higher health economic costs), and a greater disease burden than those with symptoms in only one or two parts of the day. Although previous studies have identified aspects of the disease burden associated with symptoms at specific times of day, our findings suggest that the distribution of symptoms throughout multiple parts of the day also has a significant impact on multiple outcomes. Clearer recognition of symptom burden and an individualized treatment approach may be warranted for patients who experience persistent COPD symptoms, especially for those experiencing morning, daytime, and nighttime symptoms. 


\section{Data-sharing statement}

The data that support the findings of this study are available from Adelphi Real World, but restrictions apply to the availability of these data, which were used under license for the current study, and so are not publicly available. Data are, however, available from the authors upon reasonable request and with permission of Adelphi Real World.

\section{Acknowledgments}

The authors would like to thank James Bailey for his assistance in this study. The authors thank Nina Divorty, $\mathrm{PhD}$, of Complete Medical Communications, Glasgow, UK, for providing medical writing support, which was funded by AstraZeneca, Cambridge, UK, in accordance with Good Publication Practice (GPP3) guidelines (Battisti WP, et al. Ann Intern Med. 2015;163:461-464). Some data included in this manuscript have previously been presented at the British Thoracic Society (BTS) Winter Meeting 2016, London, UK (Muñoz A, et al. Thorax. 2016;71:A202), and at the American Thoracic Society (ATS) International Conference 2017, Washington, DC, USA (Muñoz A, et al. Am J Respir Crit Care Med. 2017;195:A3669). This analysis was funded by AstraZeneca PLC. Employees of the sponsor contributed to the conception and design of the analyses, interpretation of the data, and the drafting of the manuscript.

\section{Author contributions}

All authors contributed to the conception and design of the analyses. RW and MS provided access to the data and performed the analyses. All authors contributed to the interpretation of the data and the drafting and revising of the paper and agree to be accountable for all aspects of the work. All authors approved the final manuscript for submission.

\section{Disclosure}

AM and AR were employees of AstraZeneca PLC, Barcelona, Spain at the time of the study. MS and RW are employees of Adelphi Real World. JN is an employee of AstraZeneca PLC, Madrid, Spain. The authors report no other conflicts of interest in this work.

\section{References}

1. Miravitlles M, Worth H, Soler Cataluna JJ, et al. Observational study to characterise 24-hour COPD symptoms and their relationship with patient-reported outcomes: results from the ASSESS study. Respir Res. 2014;15:122.

2. Partridge MR, Karlsson N, Small IR. Patient insight into the impact of chronic obstructive pulmonary disease in the morning: an internet survey. Curr Med Res Opin. 2009;25(8):2043-2048.
3. Global Initiative for Chronic Obstructive Lung Disease [homepage on the Internet]. Global strategy for the diagnosis, management and prevention of COPD, Global Initiative for Chronic Obstructive Lung Disease (GOLD). 2011. Available from: http://www.goldcopd.com. Accessed February 14, 2013.

4. Global Initiative for Chronic Obstructive Lung Disease [webpage on the Internet]. Global strategy for the diagnosis, management, and prevention of chronic obstructive pulmonary disease. 2017. Available from: http://goldcopd.org/gold-2017-global-strategy-diagnosis-managementprevention-copd/. Accessed March 1, 2017.

5. Espinosa de los Monteros MJ, Peña C, Soto Hurtado EJ, Jareño J, Miravitlles M. Variability of respiratory symptoms in severe COPD. Arch Bronconeumol. 2012;48(1):3-7.

6. Kessler R, Partridge MR, Miravitlles M, et al. Symptom variability in patients with severe COPD: a pan-European cross-sectional study Eur Respir J. 2011;37(2):264-272.

7. Stephenson JS, Cai Q, Mocarski M, Tan H, Doshi JA, Sullivan SD Impact and factors associated with nighttime and early morning symptoms among patients with chronic obstructive pulmonary disease. Int $J$ Chron Obstruct Pulmon Dis. 2015;10:577-586.

8. Roche N, Small M, Broomfield S, Higgins V, Pollard R. Real world COPD: association of morning symptoms with clinical and patient reported outcomes. COPD. 2013;10(6):679-686.

9. van Buul AR, Kasteleyn MJ, Chavannes NH, Taube C. Association between morning symptoms and physical activity in COPD: a systematic review. Eur Respir Rev. 2017;26(143):160033.

10. Troosters $\mathrm{T}$, van der Molen T, Polkey M, et al. Improving physical activity in COPD: towards a new paradigm. Respir Res. 2013;14:115.

11. van Remoortel H, Hornikx M, Demeyer H, et al. Daily physical activity in subjects with newly diagnosed COPD. Thorax. 2013;68(10):962-963.

12. Garcia-Aymerich J, Lange P, Benet M, Schnohr P, Antó JM. Regular physical activity modifies smoking-related lung function decline and reduces risk of chronic obstructive pulmonary disease: a population-based cohort study. Am J Respir Crit Care Med. 2007;175(5):458-463.

13. Lange P, Marott JL, Vestbo J, Nordestgaard BG. Prevalence of nighttime dyspnoea in COPD and its implications for prognosis. Eur Respir J. 2014;43(6):1590-1598.

14. Omachi TA, Blanc PD, Claman DM, et al. Disturbed sleep among COPD patients is longitudinally associated with mortality and adverse COPD outcomes. Sleep Med. 2012;13(5):476-483.

15. Anderson P, Benford M, Harris N, Karavali M, Piercy J. Real-world physician and patient behaviour across countries: Disease-Specific Programmes-a means to understand. Curr Med Res Opin. 2008;24(11): 3063-3072.

16. Reilly MC, Zbrozek AS, Dukes EM. The validity and reproducibility of a work productivity and activity impairment instrument. Pharmacoeconomics. 1993;4(5):353-365.

17. Jones PW, Harding G, Berry P, Wiklund I, Chen WH, Kline Leidy N. Development and first validation of the COPD Assessment Test Eur Respir J. 2009;34(3):648-654.

18. Rabin R, de Charro F. EQ-5D: a measure of health status from the EuroQol Group. Ann Med. 2001;33(5):337-343.

19. Jenkins CD, Stanton BA, Niemcryk SJ, Rose RM. A scale for the estimation of sleep problems in clinical research. $J$ Clin Epidemiol. 1988;41(4):313-321.

20. Miravitlles M, Worth H, Soler-Cataluna JJ, et al. The relationship between 24-hour symptoms and COPD exacerbations and healthcare resource use: results from an observational study (ASSESS). COPD. 2016;13(5): 561-568.

21. Ding B, DiBonaventura M, Karlsson N, Bergström G, Holmgren U. A cross-sectional assessment of the burden of COPD symptoms in the US and Europe using the National Health and Wellness Survey. Int $J$ Chron Obstruct Pulmon Dis. 2017;12:529-539.

22. Ding B, Small M, Bergström G, Holmgren U. COPD symptom burden: impact on health care resource utilization, and work and activity impairment. Int J Chron Obstruct Pulmon Dis. 2017;12:677-689. 
23. O'Hagan P, Chavannes NH. The impact of morning symptoms on daily activities in chronic obstructive pulmonary disease. Curr Med Res Opin. 2014;30:301-314.

24. Ding B, Small M, Bergström G, Holmgren U. A cross-sectional survey of night-time symptoms and impact of sleep disturbance on symptoms and health status in patients with COPD. Int J Chron Obstruct Pulmon Dis. 2017;12:589-599.

25. Nunes DM, Mota RM, de Pontes Neto OL, Pereira ED, de Bruin VM, de Bruin PF. Impaired sleep reduces quality of life in chronic obstructive pulmonary disease. Lung. 2009;187(3):159-163.
26. Scharf SM, Maimon N, Simon-Tuval T, Bernhard-Scharf BJ, Reuveni H, Tarasiuk A. Sleep quality predicts quality of life in chronic obstructive pulmonary disease. Int J Chron Obstruct Pulmon Dis. 2010;6:1-12.

27. Spina G, Spruit MA, Alison J, et al. Analysis of nocturnal actigraphic sleep measures in patients with COPD and their association with daytime physical activity. Thorax. 2017;72(8):694-701.

\section{Publish your work in this journal}

The International Journal of COPD is an international, peer-reviewed journal of therapeutics and pharmacology focusing on concise rapid reporting of clinical studies and reviews in COPD. Special focus is given to the pathophysiological processes underlying the disease, intervention programs, patient focused education, and self management protocols.

\section{Dovepress}

This journal is indexed on PubMed Central, MedLine and CAS. The manuscript management system is completely online and includes a very quick and fair peer-review system, which is all easy to use. Visit http://www.dovepress.com/testimonials.php to read real quotes from published authors. 